\title{
Thermoelastic Stress analysis of fatigue specimen by external lock-in signal
}

\author{
by K. S. Kim, D. S. Kim*, H. S. Chang ${ }^{\star \star \star}$, D. P. Hong ${ }^{\star \star \star \star}$ and H. C. Jung \\ *Dept. of Mechanical Design Eng., Chosun Univ., 375 Seosuk dong, Dong-Gu, Gwangju, Korea, \\ gsckim@chosun.ac.kr, yonggaryjung@gmail.com \\ *`Dept. of Advanced parts and Materials Eng., Graduate School, Chosun Univ., \\ 375 Seoseok Dong, Dong-Gu, Gwangju, Korea, victoryehdtn@naver.com \\ **Division of Precision Mechanical Eng., Chonbuk National Univ., 664-14 1Ga Duckjin-Dong, Duckjin-Gu, \\ Jeonju, Jeonbuk, 561-756, Korea, hongdp@jbnu.ac.kr \\ ***^Laser Center, Chosun Univ., 375 Seoseok Dong, Dong-Gu, Gwangju, Korea, hschang@chosun.ac.kr
}

\begin{abstract}
Fatigue behavior is strongly affected by the environment, material, and loading conditions. This paper aims to describe thermal stress measurement using Lock-in thermography as a non-destructive testing method with real time and non-contact technology. This paper measures the synchronized stress to vibration signal of actual object using the lock-in infrared thermography. Usually, lock-in infrared thermography measures the Thermoelastic stress synchronize to signal applied directly, but object is different for each the signal by properties. Thus this paper measure and evaluate it by comparing synchronized method signal of Fatigue testing machine and method synchronize extracted signal to object directly.
\end{abstract}

\section{Introduction}

Stress measurement of infrared thermography by Lock-in method is possible for measuring a temperature evolution of object by thermoelastic effect accept for the external factors. The thermoelastic effect is to the thermodynamic relationship between the change of stress in a component under elastic loading and the corresponding change of temperature. It is simply proportional to the change in the sum of the principal stresses, if adiabatic conditions fulfill. The relationship between thermal stress and strain in an isotropic, elastic element is given by the thermodynamic equation. Temperature changes in thermodynamic equation produced by changes in the applied stress. In other words, the change of temperature is proportional to the change in the sum of the principal stresses. Stress analysis of structure under dynamic loading has many advantages of infrared thermography such as non contact, whole field; insensitivity of environmental vibration enables its user to measure the stress of small and micro structure, without shape restriction.

\section{Theory}

Basically, in lock-in analysis the loading signal induced from the fatigue testing machine has to be calibrated (for each frequency) to make the temperature waveform of the loading signal truly sinusoidal. The IR camera picks up a series of thermal images and compares temperatures by extracting a sinusoidal wave pattern at each pixel of the image as shown in Fig. 1. Lock-in method is similar to a least mean square method or a single term Fourier series. For performing Lock-in method, IR image of at least 4 equidistant phase positions in the Lock-in period are necessary, which according to the Nyquist sampling theory are 2 samples for each phase component (in- phase and out-of-phase or quadrature component).

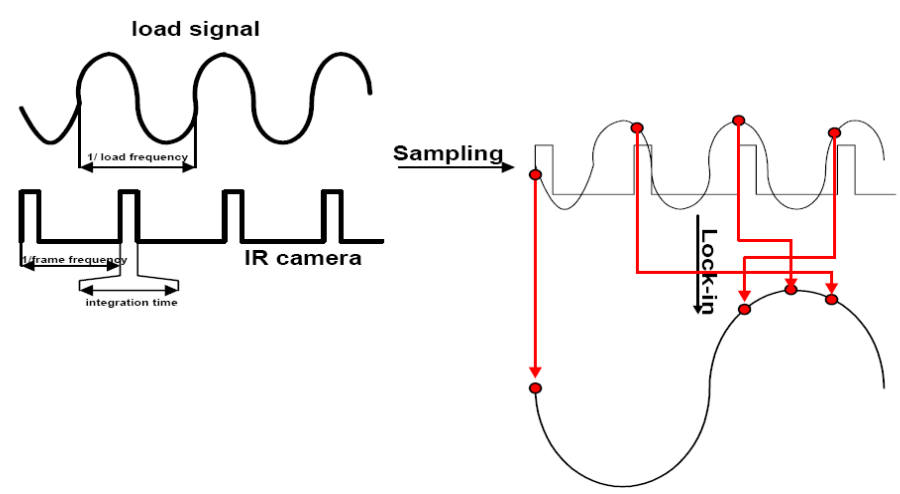

Fig. 1. Signal processing of lock-in infrared thermography 
If these image captured within a single lock-in period, this leads to an upper limit for the lock-in frequency of floading =frame /4. This case is called "4-point correlation"

$$
\text { Signals }(x, y)=c \sum_{i=0}^{N} F_{i} T_{i}(x, y)
$$

where $\mathrm{c}$ is a coefficient, $\mathrm{Fi}$ is a weighting factor, $\mathrm{Ti}(\mathrm{x}, \mathrm{y})$ is the temperature of pixel $(\mathrm{x}, \mathrm{y})$, $\mathrm{Fi}$ is calculated by a real time correlator. Signals are results of real time processing. The signal is mixed with the reference signal and integrated over the time. Typically, the reference signals are cleaned up and normalized in digital electronics processing. This algorithm will find the correlation between the dynamic thermal signal $\mathrm{Ti}(\mathrm{x}, \mathrm{y})$ and the dynamic reference signal $\mathrm{Fi}$. This equation results from subtraction of the original image from the shifted image by the shearing magnitude certain number of pixels along the $x$ or $y$ axis.

\section{Experiment and Result}

In order to measure thermoelastic stress should be applied Phase-locking techniques. The displacement/acceleration through measurement sensor for structure is measured a change in the status of dynamic loads. Then signal extraction and mid-infrared thermography camera is synchronized. So, the forecasting system is configured. At this point, a nonlinear load of the interval using dynamic loading system is applied. And real-time will be obtained a linear/nonlinear signal acquisition using displacement/acceleration sensor. The acquired signals are measured with the synchronized infrared thermography for thermoelastic stress of object's.
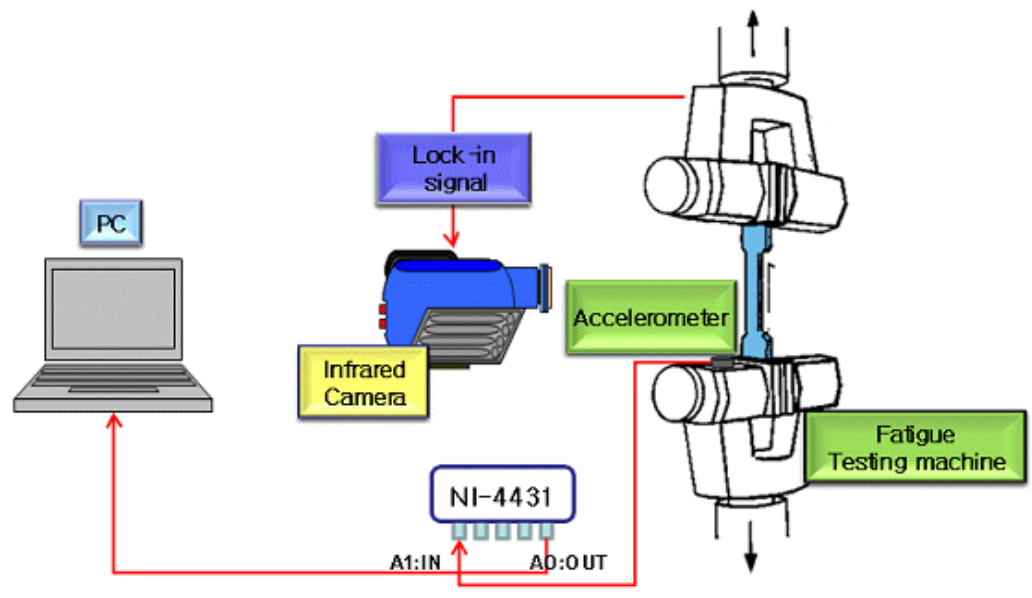

Fig. 2. Signal processing of lock-in infrared thermography

\section{REFERENCES}

[1] Rauch B J and Rowlands R E, Thermoelastic stress analysis in Handbook on experimental mechanics ed by A.S. Kobayashi, SEM , 1993.

[2] Bremond $\mathrm{P}$ and Potet $\mathrm{P}$, Lock-In Thermography: A tool to analyse and locate thermo-mechanical mechanisms in materials and structures, Thermosense XXII, 2001.

[3] Awrejcewicz, J., Pyryev, Y, "Thermoelastic contact of a rotating shaft with a rigid bush in conditions of bush wear and stick-slip movements", International Journal of Engineering Science, 2002 40(10) 1113-1130.

[4] Luong M P, Fatigue limit evaluation of metals using an infrared thermographic technique, Mechanics of Materials, 1998. 